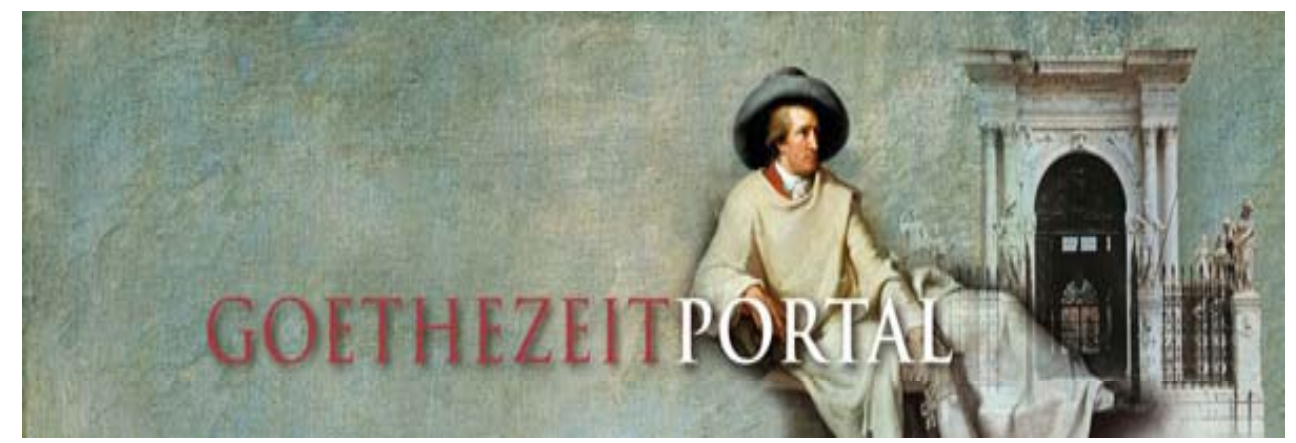

MALTE STEIN

\title{
Johann Wolfgang Goethe: „Harzreise im Winter“
}

Erstpublikation:

Jörg Schönert / Peter Hühn / Malte Stein: Lyrik und Narratologie. Text-Analysen zu deutschsprachigen Gedichten vom 16. bis zum 20. Jahrhundert (Narratologia. Contributions to Narrative Theory; 11) Berlin: de Gruyter 2007, S. 75-97.

Vorlage:

PDF-Datei des Autors.

Autor:

Dr. Malte Stein

Hansa-Kolleg

Von-Essen-Str. 82-84

22081 Hamburg

E-Mail:<stein_malte@yahoo.de> 
Malte Stein

\section{Johann Wolfgang Goethe: „Harzreise im Winter“}

(I)

$\begin{array}{ll} & \text { Dem Geier gleich, } \\ & \text { Der auf schweren Morgenwolken } \\ \text { Mit sanftem Fittich ruhend } & \text { Nach Beute schaut, } \\ & \text { Schwebe mein Lied. }\end{array}$

(II)

Denn ein Gott hat Jedem seine Bahn Vorgezeichnet, Die der Glückliche

10 Rasch zum freudigen

Ziele rennt:

Wem aber Unglück

Das Herz zusammenzog,

Er sträubt vergebens

15 Sich gegen die Schranken

Des ehrenen Fadens

Den die doch bittre Schere

Nur einmal löst.

(III)

20

In Dickichts Schauer

Drängt sich das rauhe Wild,

Und mit den Sperlingen

Haben längst die Reichen

In ihre Sümpfe sich gesenkt.

(IV)

25

Leicht ists folgen dem Wagen

Den Fortuna führt,

Wie der gemächliche Troß

Auf gebesserten Wegen

Hinter des Fürsten Einzug.

(V)

Aber abseits wer ists?

Ins Gebüsch verliert sich sein Pfad,

Hinter ihm schlagen

Die Sträuche zusammen

Das Gras steht wieder auf,

Die Öde verschlingt ihn.

(VI)

35 Ach wer heilet die Schmerzen

Des, dem Balsam zu Gift ward?

Der sich Menschenhaß 
Aus der Fülle der Liebe trank, Erst verachtet, nun ein Verächter, Zehrt er heimlich auf Seinen eignen Wert In ungnügender Selbstsucht.

(VII)

Ist auf deinem Psalter,

Vater der Liebe, ein Ton

Seinem Ohre vernehmlich, So erquicke sein Herz! Öffne den umwölkten Blick Über die tausend Quellen Neben dem Durstenden In der Wüste.

(VIII)

Der du der Freuden viel schaffst, Jedem ein überfließend Maß, Segne die Brüder der Jagd Auf der Fährte des Wilds

55 Mit jugendlichem Übermut Fröhlicher Mordsucht, Späte Rächer des Unbills, Dem schon Jahre vergeblich Wehrt mit Knütteln der Bauer.

(IX)

60 Aber den einsamen hüll

In deine Goldwolken, Umgib mit Wintergrün, Bis die Rose wieder heranreift Die feuchten Haare,

65 O Liebe, deines Dichters!

(X)

Mit der dämmernden Fackel Leuchtest du ihm Durch die Furten bei Nacht, Über grundlose Wege

70 Auf öden Gefilden; Mit dem tausendfarbigen Morgen Lachst du ins Herz ihm, Mit dem beizenden Sturm Trägst du ihn hoch empor.

75 Winterströme stürzen vom Felsen In seine Psalmen,

Und Altar des lieblichsten Danks

Wird ihm des gefürchteten Gipfels 
Du stehst mit unerforschtem Busen

Geheimnisvoll offenbar

Über der erstaunten Welt,

85 Und schaust aus Wolken

Auf ihre Reiche und Herrlichkeit,

Die du aus den Adern deiner Brüder

Neben dir wässerst.

Johann Wolfgang Goethe: Sämtliche Werke nach Epochen seines Schaffens (Münchner Ausgabe) Bd. 2.1. München 1987, S. 37-41.

\section{Biographischer Hintergrund}

Bekanntlich ist bereits der Entstehungskontext des berühmten Hymnus so geheimnisvoll wie erzählenswert:

In schlechter Jahreszeit machte sich Goethe am 29. November 1777 zu einer Reise auf, die Merkmale des Besonderen trug. Der Herzog [Carl August von Sachsen-Weimar] hatte sich zwei Tage zuvor zur Jagd ins Eisenachische begeben, während Goethe ,nach einem kleinen Umweg“" nachkommen wollte. Wohin ihn dieser Umweg führen sollte, darüber schwieg er gegenüber jedermann. In seinem Tagebuch stand schon unter dem 16. November: „Projekte zur heimlichen Reise“. Auf der Reise selbst bezeichnete er sein geheimgehaltenes Unternehmen als „Wallfahrt“ (an Charlotte von Stein, 7.12.1777). Es zog ihn in den Harz. [...] Von Süden nach Norden durchquerte der Reiter das Gebirge, besichtigte die Baumannshöhle [...], ritt am 4. Dezember nördlich am Harz entlang bis Goslar und nahm das Winterwetter ruhig in Kauf. [...] In den nächsten Tagen Besuch des Harzer Bergwerksreviers, Einstieg in verschiedene Gruben und dann am 10. Dezember der Höhepunkt [der sich im Tagebuch wie folgt festgehalten findet]: „Früh nach dem Torfhause in tiefem Schnee. 1 viertel nach 10 aufgebrochen von da auf den Brocken. Schnee eine Elle tief, der aber trug. 1 viertel nach eins droben. heitrer herrlicher Augenblick, die ganze Welt in Wolcken und Nebel und oben alles heiter. Was ist der Mensch dass du sein gedenckst [...].“1

Wie aus Tagebucheintragungen und brieflichen Äußerungen hervorgeht, hat Goethe den Verlauf seiner ersten Harzreise mitsamt der zur Winterszeit damals unüblichen Brockentour als eine Art Zeichen gedeutet, über dessen Sinngehalt er sich jedoch in seinen biographischen Zeugnissen ausschwieg. „Man weiß, daß Goethe seiner Besteigung des Brockens vorher und auch nachher eine Bedeutung zugemessen haben muß, die sie

1 Conrady (1987), S. 370 (Ergänzungen in eckigen Klammern von MS). 
auch als immerhin beachtliche alpinistische Leistung [per se, MS] nicht gehabt hat; aber man weiß nicht, welche Bedeutung das war. ${ }^{\text {"2 }}$ Vor diesem Hintergrund ist wiederholt versucht worden, das während der ominösen Reise zumindest schon begonnene Gedicht „Harzreise im Winter“ als „Auskunftmittel“ ${ }^{13}$ zu nutzen, wobei man sich allerdings zweier grundlegend verschiedener Vorgehensweisen bediente: Während es die Mehrheit der Kommentatoren für legitim und sinnvoll erachtet hat, erläuterungsbedürftige Textelemente aus dem biographischen Kontext heraus zu erklären, unterstellen Weimar und Wellbery in zwei miteinander konkurrierenden „Harzreise“-Analysen, dass der publizierte Gedichttext als ÄuBerung in einer öffentlichen Kommunikation auch ohne biographische Kenntnisse verstehbar sein müsse - er auf Seiten der Leserschaft lediglich ein gewisses kulturelles Wissen voraussetze.

David Wellbery zieht aus dieser Prämisse die Konsequenz, auf Bezüge zur Biographie gänzlich zu verzichten. Dem postmodernen Intertextualitätskonzept verpflichtet, schreibt er dem Gedicht eine „kulturelle Dimensionalität“ zu, die angeblich ,an kein einzelnes biographisches Subjekt rückzubinden ist" ${ }^{4}{ }^{4}$ Klaus Weimar dagegen vertritt die Ansicht, dass aus dem Textsinn, der im Rahmen einer biographie-unabhängigen Analyse zu ermitteln sei, auf die Bedeutung der Reise für Goethe durchaus geschlossen werden dürfe. Weimars Einschätzung zufolge sind die „Bilder des Textes biographisch instrumental“ in dem Sinne, dass sie als „Instrumente zur Deutung des Weges“ dienen, „den ihr Autor biographisch gegangen ist". 5

Inwiefern Letzteres zutrifft, vermag auch die folgende Analyse nicht zu entscheiden, da Aussagen zur Autor-Biographie ihren Untersuchungsbereich überschreiten. ${ }^{6}$ Im Rückgriff auf narratologische Begriffe wird hier aber gleichwohl eine neue Lesart des enigmatischen Gedichts entwickelt, die, wenn man sie denn tatsächlich als des reisenden Goethe ,confessio“ annähme, der biographisch verbürgten „Wallfahrt" ${ }^{\text {"7 }}$ eine erstaunlich häretische Bedeutung verliehe.

2 Weimar (1984), S. 16

3 Ebd., S. 17.

4 Wellbery (1984), S. 80.

5 Weimar (1984), S. 35.

6 Eine gute Sammlung und anregende Interpretation der biographischen Zeugnisse aus dem Umkreis von Goethes erster Harzreise bietet Dwars (1998).

7 Goethe an Charlotte von Stein vom 7.12.1777, zitiert nach Conrady (1987), S. 370. 


\section{Rezeptionslenkende Funktion der Überschrift}

Durch die Gedichtüberschrift „Harzreise im Winter“ werden beim Leser sogleich zwei Schemata aufgerufen; zum Einen der Frame ,Harzlandschaft im Winter', eine Szenerie, die als unabdingbare (Kern-)Elemente die Gegebenheiten ,Berge' und ,Schnee' umfasst; zum Andern das Skript ,Reise', das sich in seiner abstraktesten Form aus den Sequenzelementen ,Aufbruch', ,Zurücklegen einer Wegstrecke' und ,Ankunft' (bzw. auch ,Nicht-Ankunft') zusammensetzt.

Die Kombination dieser beiden Schemata weckt (mindestens) die Erwartung, dass in Goethes Gedicht der Verlauf einer Reise dargestellt wird, deren durch ein winterliches Gebirge führende Wegstrecke etappenweise mit besonderen Anstrengungen und Gefahren verbunden sein kann.

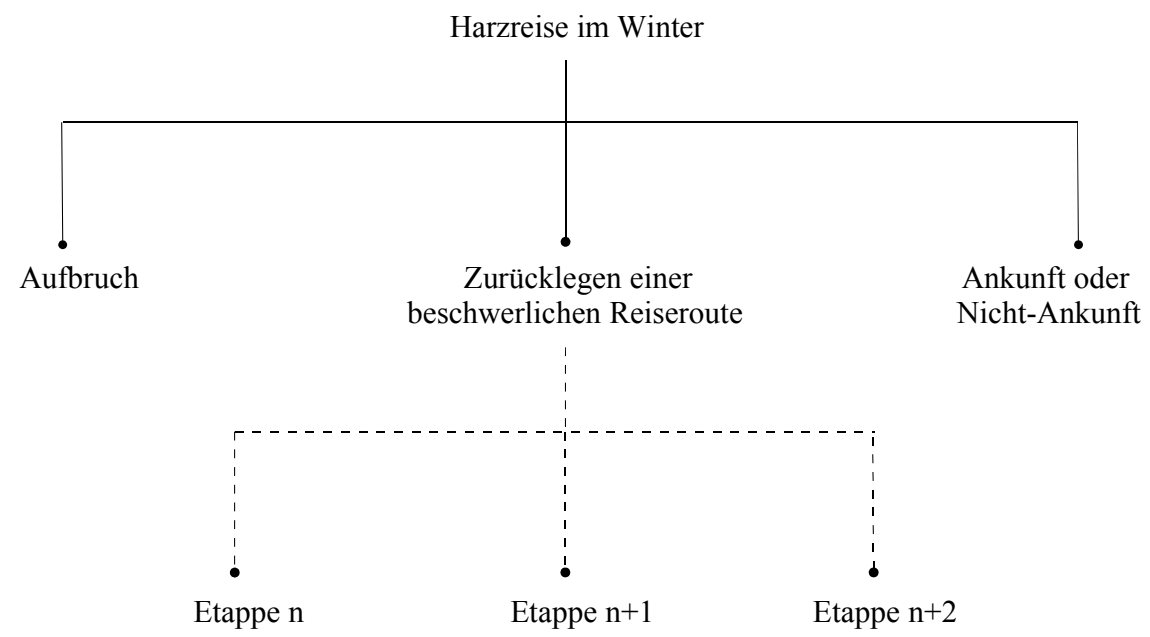

Abb. 1: Sequenzschema A

Dieses auf pragmatischen Präsuppositionen beruhende Sequenzschema wird noch um eine Ebene komplexer, wenn man berücksichtigt, dass Reisen ein zielgerichtetes Handeln ist, zu dem sich jemand aus irgendeiner Veranlassung und in irgendeiner Absicht entschlossen haben muss. 


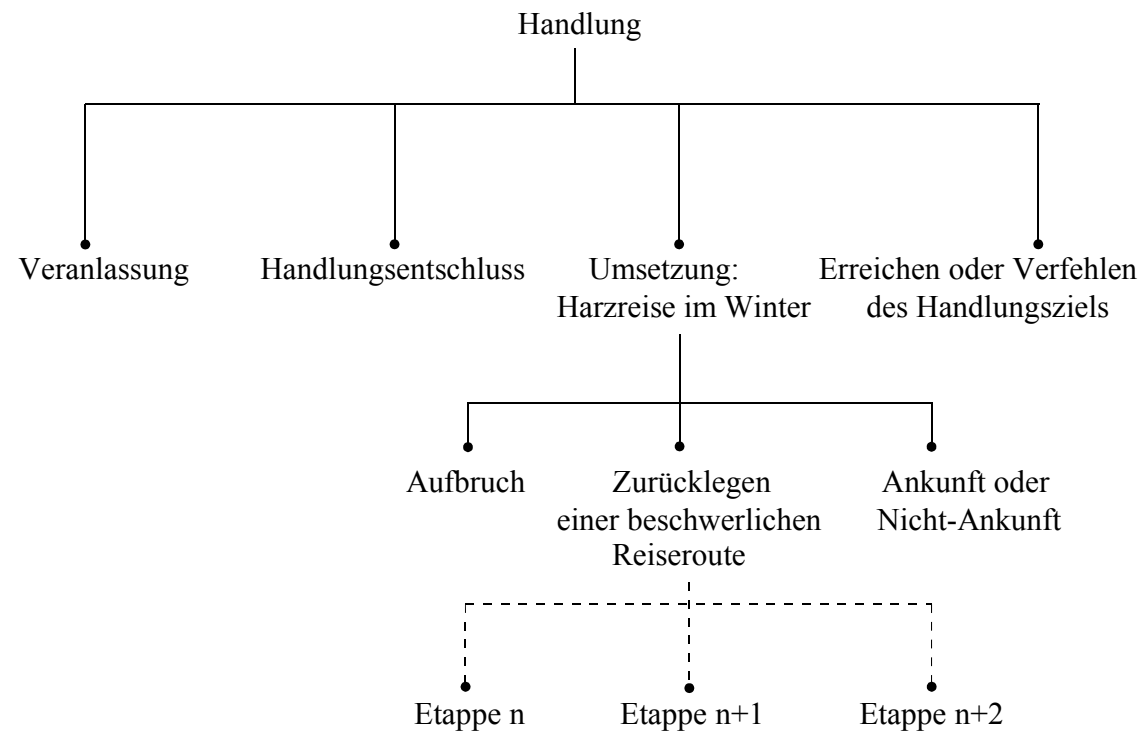

Abb. 2: Sequenzschema B

Nach Kenntnisnahme der Überschrift hat der Leser also bereits - mehr oder minder reflektiert - gewisse Strukturhypothesen in Bezug auf das dargestellte ,Was'. Durch den Gedichttitel vorbereitet, nimmt er die Lektüre (oder auch Analyse) des Textes mit vorläufigen Schemata auf, die bestimmte Informationen erwarten lassen und es ermöglichen, das im Text sukzessiv dargebotene ,Einzelne' versuchsweise in einen unterstellten Gesamtzusammenhang einzuordnen. ${ }^{8}$

8 Obwohl schon der Titel „Harzreise im Winter“ „einen gewissen Grad der Narrativierung erwarten läßt" (Wellbery [1984], S. 59), haben sich die bisherigen Interpreten des Textes entweder gar nicht oder nicht hinlänglich darum bemüht, die im Text dargebotenen Geschehenselemente zu Handlungssequenzen zu ordnen. Weimar beispielsweise umgeht das Problem der Sequenzen-Rekonstruktion, indem er sich darauf beschränkt, die Kohärenz des dargestellten ,Was' fast ausschließlich auf der Ebene von Sem- und Bildrekurrenzen aufzuzeigen. Dagegen führt Wellbery bereits aus, dass als kohärenzbildende Faktoren neben semantischen Isotopien auch kognitive Schemata wirksam sind, welche dem Rezipienten, sobald er sie auf bestimmte Textsignale hin aktiviert, als konzeptuelle Basis für die Feststellung von impliziten Geschehenszusammenhängen dienen. Zur Liste der für die „Harzreise“-Lektüre besonders wichtigen Schemata zählt Wellbery dann allerdings mit dem Skript ,Jagd“ (neben ,Dichtung“, ,Weg', ,Flüssigkeit', ,Wahrnehmung' und ,Religion') nur ein einziges Sequenzschema (S. 53), so dass die Sequenzanalyse auch bei ihm noch unterentwickelt bleibt. 


\section{Konkretisierung der durch die Überschrift aufgerufenen Schemata}

Auf Geschehenselemente und Gegebenheiten, die sich als Teile der angekündigten Szenerie begreifen lassen, stößt der Leser schon in den ersten Strophen (I u. III): Der nach Beute suchende Raubvogel (V.1: „Geier“), das im Unterstand (V.19: „Schauer“) sich drängende Pelzwild, die nach tradierter Vorstellung in den Sümpfen überwinternden Spatzen sowie das Vergrabensein auch der „Reichen“ (V.22) in ihren Winterquartieren - das alles passt bereits ins Bild eines winterlichen Harzes und wird in den Strophen V, VIII und IX bis XII durch zahlreiche weitere Elemente noch ergänzt (V.30: „Gebüsch“, „Pfad“, V.32: „Sträuche“, V.33: „Gras“, V.34: „Öde“, V.62: „Wintergrün“, V.68: „Furten bei Nacht“, V.69: „grundlose Wege“, V.70: „öde Gefilde“, V.71: „tausendfarbiger Morgen“, V.73: „,beizender Sturm“,V.75: „Winterströme“, „Felsen“, V.78: „gefürchteter Gipfel“, V.79: „schneebehangener Scheitel“, V.85: „Wolken“).

Auf sich warten lässt dagegen bis Strophe V ein erstes Geschehnis, das als Sequenzelement einer „Harzreise identifiziert werden kann. Von einer Reise zwar ist in der vierten Strophe schon die Rede, wo sich der „Troß“ (V.26) und der „Einzug“ (V.28) des Fürsten erwähnt finden. Sofern dort aber an den „Einzug“ ins Winterquartier zu denken ist - der Fürst und seine Gefolgsleute zählen zu den „Reichen“, die sich zum Zeitpunkt der Sprecher-Rede „längst“ (V.22) schon in ihre „Sümpfe“ (V.23) (= Winterunterkünfte) ,gesenkt“ (V.23) haben -, muss der ,auf gebesserten Wegen“" (V.27) vollzogene Umzug noch vor dem Winter erfolgt sein. Tatsächlich zur Winterszeit unterwegs sind erst (a) der Wanderer „abseits“ (V.29), dessen „Pfad“ sich ,ins Gebüsch verliert“ (V.30), und (b) der durch Äquivalenzbeziehung mit diesem eng verbundene „Dichter“ (V.65), der auf abenteuerlicher Route - erst nämlich „durch die Furten bei Nacht" (V.68) und dann ,über grundlose Wege auf öden Gefilden“ „hoch empor" (V.74) auf den Gipfel des Brocken gelangt.

Angedeutet werden somit gleich zwei Winterreise-Verläufe, die sich dem Anschein nach zueinander in Gegensatz befinden: Während die Darstellung des einen Verlaufs mit dem Verschwinden oder gar der Vernichtung des Wanderers endet - „die Öde verschlingt ihn“ (V.34) -, bricht die Darstellung des anderen mit dem Erreichen eines Etappenziels ab. 

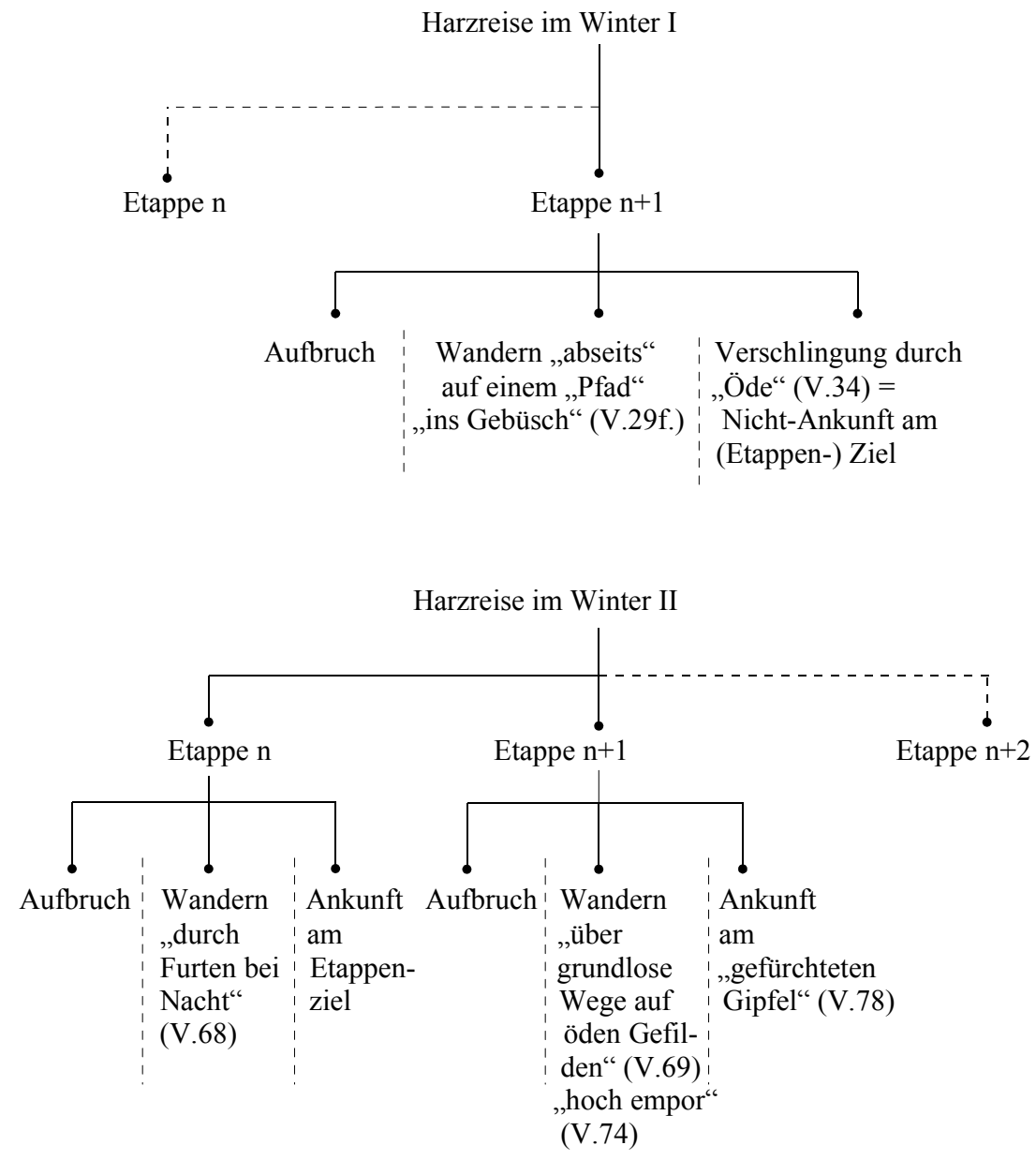

Abb. 3: Sequenzen A

\section{Semantisierung der Reise als Symbol eines besonderen Lebenslaufs}

Bevor ab der fünften Strophe die eigentliche Darbietung der doppelten ,Harzreise im Winter' beginnt, wird das Reise-Skript zunächst als ein komplexes Symbol codiert. Beim Lesen der Strophen II und IV kommt es zu einer assoziativen Verschmelzung der durch die Überschrift aktivierten Schemata mit einer auf die antike Mythologie anspielenden Lebenslauf- 
Metaphorik: Der winterliche Harz erhält die Konnotation, ein Bild für die allgemeine Beschaffenheit der Welt zu sein; der abseitige und gefahrvolle Reiseweg erscheint als göttlich vorherbestimmte ,Lebensbahn'.

\section{Klassifikation der Figuren}

Aus den Strophen II und IV mit ihren als allgemeingültig formulierten Aussagen ergibt sich eine Art Grundordnung der erzählten Welt. Der Leser kann diesen Strophen entnehmen, (1) dass in der erzählten Welt mindestens „ein Gott" (V.6) und Nicht-Götter (sterbliche Lebewesen) existieren; (2) dass besagter „Gott“ einem jeden Lebewesen dessen Lebensweg „Vorgezeichnet“ (V.8) hat; (3) dass sich die Menge der Lebewesen in "Glückliche“ (V.9) und Unglückliche unterteilt; (4) dass ein "Glücklicher" der ist, der seiner ihm vorgegebenen „Bahn“ (V.7), insofern sie „auf gebesserten Wegen“ (V.27) zu einem „freudigen Ziele“ (V.10f.) führt, „leicht“ (V.24) folgen kann; (5) dass sich hingegen derjenige, dem „Unglück das Herz zusammenzog“ (V.12f.) - dessen Lebensbahn von „Fortuna[s]“ (V.25) Wagenspur abweicht -, „vergebens“ (V.14) gegen sein Schicksal sträubt.

Als ein weiteres Strukturraster intratextuell etabliert ist mit diesen Propositionen ein Schema zur (wiederum versuchsweisen) Klassifizierung der im Gedicht dargestellten Figuren:

\begin{tabular}{|c|c|}
\hline \multicolumn{2}{|c|}{ Gottheit(en) } \\
\hline \multicolumn{2}{|c|}{ Sterbliche Lebewesen } \\
\hline \multicolumn{2}{|c|}{ mit der Bestimmung, } \\
mit der Bestimmung, \\
einen leichten Lebenslauf \\
einen schweren Lebenslauf \\
auf unbefestigten Wegen \\
zu haben \\
$=$ \\
$\begin{array}{c}\text { zu haben } \\
\text { Glückliche, die ihrer Bestimmung } \\
\text { willig folgen }\end{array}$ \\
Unglückliche, die sich gegen ihre \\
Bestimmung erfolglos sträuben
\end{tabular}

Abb. 4: Figurenklassen

Das Leben der Glücklichen veranschaulicht die Art und Weise, in der das „rauhe Wild“ (V.20), die „Sperlinge“ (V.21) und die „Reichen“ (V.22) über den Winter kommen. Deren in Strophe III beschriebenes Winterleben, das gekennzeichnet ist durch die Merkmale (1) ,Behaustsein“ 
(V.19: „Schauer“, V.23: „Sümpfe“), (2) ,Gemeinschaft“ (V.20: „drängen“, Nennung im Plural) und (3) ,Prokreation“ (Sperling als Vogel der Aphrodite, konnotiert mit Vermehrungslust; Sumpf als Pfuhl, konnotiert mit Sünde / Sexualität), wird mit der vierten Strophe in die aus der zweiten schon vertraute Wegmethaphorik übersetzt: Wer ein derartiges Leben führt - so besagt Strophe IV als ein nachträglicher Kommentar zu III -, der zieht auf bequemen Wegen hinter Fortunas Wagen her und vermag deshalb sein Schicksal bereitwillig anzunehmen.

Was es hingegen bedeutet, einer Bahn „abseits“ (V.29) der GlücksWege folgen zu müssen, ist mit dem Winterreisenden aus den Strophen V bis VII und IX dargestellt. Als ein zu einsamer Wanderschaft Bestimmter, der im Gegensatz zu den vorher genannten Figuren (1) ohne Behausung, (2) ohne Gemeinschaft und (somit) (3) ohne Nachkommen bleibt, hinterlässt er auf seinem Lebensweg keinerlei Spuren: „Hinter ihm schlagen die Sträuche zusammen, / Das Gras steht wieder auf, / Die Öde verschlingt" (V.31-34) nicht etwa nur ihn selber, sondern im gleichen Moment auch seine spärlichen Lebenszeugnisse.

Es führt die Lebensbahn dieses „[E]insamen“ (V.60) auf das un-,,freudige Ziel“" (V.10f.) eines schnellen Vergessenseins zu - und bietet ihm deshalb Grund, sich mit der göttlichen Wegvorgabe nicht abfinden zu können. Zwar ist von einem solchem Aufbegehren nicht ausdrücklich die Rede, gewiss. Insofern aber aus Strophe VI hervorgeht, dass jener Wanderer - als „erst verachtet, nun ein Verächter“ (V.39) - ein von Leiderfahrung geprägtes Herz voll „Menschenhaß“ (V.37) hat, lässt sich auf eine Diskrepanz zwischen Wollen und Schicksal in seinem Falle doch schließen: „Wem [...] Unglück das Herz zusammenzog“ (V.13f.) wie ihm, „Er sträubt vergebens / Sich gegen die Schranken / Des ehernen Fadens“ (V.14ff.) ...

Ein entsprechender Konflikt wäre dann eigentlich auch bei dem zweiten Winterreisenden zu erwarten, da dieser ja ebenfalls unbehaust und ohne Gemeinschaft ist. Doch obwohl dessen Lebensbahn einmal mehr auf ein glückloses Dasein hindeutet - über ,grundlose Wege“ (V.69) auf einen verschneiten Gipfel kann Fortunas „Wagen“ (V.24) nicht rollen -, scheint der Besteiger des Brockens mit seiner Bestimmung in Einklang zu sein (was als ein Verstoß gegen die vorher angezeigte Korrelation von Abseitigkeit und Unglück den Status eines Ereignisses hat):

Durch göttliche Lenkung an einen Ort gelangt, der in größter Ödnis liegt und von ,ahndende[n] Völker[n]“ (V.81) aus „Geister“-Furcht gemieden wird, zeigt sich der Bergbezwinger durchaus erfreut über seine 
Situation. Indem er „des gefürchteten Gipfels [...] Scheitel“ (V.78f.) zum „Altar des lieblichsten Danks“ (V.77) sich macht, bringt er gegenüber der gepriesenen Gottheit zum Ausdruck, die ihm vorbestimmte Lebensbahn glücklich angenommen zu haben.

Dass ihm dies, anders als dem vorangegangenen Wanderer, möglich ist, lässt sich zunächst damit erklären, dass er am Ende trotz größter Isolation keine ,Verschlingung' (mehr) fürchten muss. In seiner Eigenschaft als „Dichter“ (V.65) nämlich hat dieser ,[E]insame“ (V.60) die ihm verwehrte Prokreation durch künstlerische Kreativität ersetzen können. Seine in der elften Strophe erwähnten „Psalmen“ (V.76) nebst jenem „Lied“ (V.5), das er - als der fiktive Sprecher des Textes - einem „Geier gleich“ (V.1) in die „Welt“ (V.84) entsendet, bieten ihm eine Gewähr dafür, dass sich die Spur seiner Erdentage vorerst nicht ,verliert“ (vgl. V.30).

\begin{tabular}{|c|c|c|}
\hline & auf „,gebesserten Wegen“(V.27) & $\begin{array}{c}\text { auf ,grundlosen Wegen“(V.69) } \\
\text { (abseits) }\end{array}$ \\
\hline Im Tal & 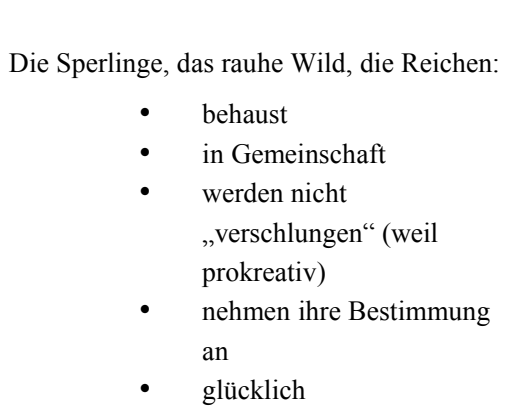 & $\begin{aligned} & \text { Der Menschen-,,Verächter“: } \\
& \text { • } \text { unbehaust } \\
& \text { • } \text { einsam } \\
& \text { • } \text { wird „,verschlungen” } \\
& \text { (weil nicht prokreativ) } \\
& \text { • } \text { sträubt sich gegen seine } \\
& \text { Bestimmung } \\
& \text { • } \text { unglücklich }\end{aligned}$ \\
\hline $\begin{array}{l}\text { Auf dem } \\
\text { Gipfel }\end{array}$ & & 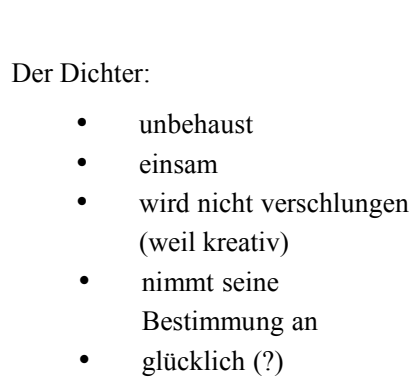 \\
\hline
\end{tabular}

Abb. 5: Semantische Oppositionen und Äquivalenzen der in die Weg-Metaphorik einbezogenen Figuren (unvollständig) 


\section{6. (Vorläufige) Bestimmung der zentralen Zustandsveränderung}

Begreift man den Menschen-,,Verächter“ (V.39) und den „Dichter“ (V.65) als eine personelle Einheit - wofür neben den diversen Äquivalenzmerkmalen auch die Tatsache spricht, dass in der neunten Strophe die Rede vom „einsamen“ zunächst auf den „Verächter“ referiert, sodann aber ganz unvermittelt zur Rede vom „Dichter“ gerät - so repräsentieren die beiden Wanderer-Darstellungen den Anfangs- und Endzustand eines Prozesses, in dessen Verlauf sich ein Glückloser mit seinem Lebenslos (bzw. dem dafür verantwortlichen Gott) aussöhnt.

Die angedeutete Brockenbesteigung wird bei solcher Betrachtung zu einem Symbol für die Überwindung einer psychischen Krise - erscheint als ,pictura' für den Aufschwung bzw. die Heilung einer von „Unglück“ (V.12) beschwerten Seele. Aus einem Tal der Melancholie aufsteigend (aus „Menschenhaß“ [V.37], „Schmerzen“ [V.35] und Selbstentwertung in ,,ungenügender Selbstsucht“ [V.42]), erreicht der „Dichter“ buchstäblich ,per aspera' eine gottähnliche Stellung ,über der erstaunten Welt" (V.84).

\section{Einordnung der Zustandsveränderung in eine übergeordnete Makrosequenz}

Im Zuge eines solchen Übergangs von den Signifikaten erster Ordnung auf die Ebene der Signifikate zweiter Ordnung verändert sich mit der Bedeutung des dargestellten Geschehens auch dessen sequentielle Struktur: Die angedeutete Besteigung des Brockens ist nur auf der ,pictura'-Ebene das Sequenz-Element einer ,Harzreise im Winter'. Sobald man die Bergtour als die bildliche Repräsentation eines Heilungspozesses entziffert, wird sie - auf der ,subscriptio'-Ebene - zum Element einer anderen Sequenz.

Bereits in den Strophen VI und VII ist durch die Terme „Gift" (V.36), „Schmerzen“ (V.35), „Balsam“ (V.36), „Ach, wer heilet“ (V.35) und ,erquicke sein Herz" (V.46) das allgemeine Skript einer (vergiftungsbedingten) ,Erkrankung ' aufgerufen worden, in das sich das Wandergeschehen nun (neben noch weiteren Signifikanten zweiter Ordnung) integrieren lässt: 


\section{Erkrankung}

- Verursachung: Trinken von „Balsam“, der als „Gift“ (V.36) wirkt: die „Fülle der Liebe“ (V.38) / Verachtung $\rightarrow$ Zusammenziehung des Herzens durch Unglück

- Symptome: „Schmerzen“ (V.35), „Menschenhaß“ (V.37), „Aufzehren“ (vgl. V.40) des „eigenen Wertes“ (V.40f.) in „ungenügender Selbstsucht“ (V.42) $\rightarrow$ Einsamkeit / Unbehaustheit ohne Prokreation $\rightarrow$ Sträuben gegen die vorgezeichnete Lebensbahn

- Hilfeappell: (durch den Sprecher): „Vater der Liebe, [...] erquicke sein Herz!“ (V.44, 46), ,öffne den umwölkten Blick“ (V.47), „,den Einsamen hüll in deine Goldwolken“(V.60f.); ,[O Liebe], umgib mit Wintergrün die feuchten Haare [...] Deines Dichters" (V.62, 64)

- Maßnahmen des Helfers: „Liebe“ (V.65) „leuchtet“ (V.67) mit „dämmernder Fackel“ (V.66) durch „Furten bei Nacht“ (V.68); führt über ,grundlose Wege“ (V.69); „trägt“ (V.74) mit dem „,beizenden Sturm“ (V.73) empor; „lacht“ (V.72) mit dem „tausendfarbigen Morgen“ (V.71) „ins Herz“ (V.72)

- Wirkung: Erreichen des Gipfels; [Erlangung von künstlerischer Produktivität]; Aufgabe des Sträubens gegen die vorgezeichnete Lebensbahn (V.1416); Überwindung von „Selbstsucht“ (V.42) und „Menschenhaß“ (V.37); Klassenwechsel zu den „Glücklichen“(V.9)

- Danksagung: Psalmengesang als „lieblichster Dank“(V.77)

Abb. 6: Schema (Skript) und Verlauf der Sequenz ,Erkrankung

Aufgrund seiner besonderen Integrationskraft - keines der in dem Gedicht sonst noch indizierten und / oder etablierten Schemata vermag eine ebenso große Menge an Propositionen aufzunehmen - darf das hier angeführte Skript als die Makro-Strukur des dargestellten Geschehens gelten. Sobald man einmal beginnt, aus Goethes Text eine Krankheits- und Heilungsgeschichte zu abstrahieren, lassen sich diesbezüglich relevante Informationen aus jeder einzelnen Strophe ableiten; nämlich

- aus Strophe VI Informationen über die ,Krankheitsursachen und -symptome',

- aus den Strophen V und II (V.12-16) in Kontrast zu den Strophen III, IV und II (V.6-11) Informationen über die Auswirkungen der ,Krank- 
heit' auf des ,Erkrankten' Leben und Zukunft (siehe oben die Ausführungen zur Klassifikation der Figuren),

- aus den Strophen VII und IX Informationen über die Hilfe-Appelle an einen offenbar heilungsmächtigen (,Arzt'-)Gott,

- aus den Strophen VIII, XI, XII und II Informationen über die (weiteren) Eigenschaften bzw. die Identität dieses Gottes,

- aus Strophe X Informationen über die Art seiner ,Heilmaßnahmen',

- aus den Strophen XI, XII und I Informationen über die ,therapeutische" Wirkung jener Maßnahmen - und somit über den Endzustand des ursprünglich ,Kranken`.

Vor allem in Bezug auf die letzten drei Punkte (die Identität des Gottes, die Art seiner Hilfe und deren Effekte) erfolgt die Informationsvergabe auf eine überaus implizite Weise, so dass hier das Maß der vom Leser zu erbringenden Erschließungsarbeit - wie sich gleich zeigen wird: aus inhaltlichen Gründen - besonders hoch ausfällt.

\section{Identität des Gottes}

Jener „Vater der Liebe“ (V.44), an den der extradiegetische Sprecher in VII appelliert, er möge den „,[E]insamen“ (V.60) von dessen „Schmerzen [heilen]" (V.35), ist in der bisherigen Rezeption des Textes zumeist als Vater der christlichen Nächstenliebe - als Gott der , $\alpha \gamma \alpha \pi \eta$ ' (Agape) identifiziert worden. ${ }^{9}$ Ausschlaggebend dafür dürften Diskursmarker wie

9 So beispielsweise von Friedrich (1991), S. 172, Weimar (1984), S. 26, und Wellbery (1984), S. 58. Wellbery unterstellt, dass der fiktive Sprecher des Gedichts in Strophe VII an einen Gott mit „entschieden judäo-christlichen Zügen“ sich wende, sodann aber in Strophe VIII eine Bitte an den ,[s]elbstverständlich“ graeco-romanischen „Gott des Weines“ richte (S. 58). Gesetzt einmal, dass diese Beschreibung zuträfe, müssten aus ihr zwei alternative Schlüsse gezogen werden: Entweder wäre nämlich der Sprechakt aus Strophe VIII als eine ereignishafte Regelverletzung zu identifizieren, insofern doch einer der Haupt-,,Züge“ des jüdisch-christlichen Gottes die Forderung ist, dass seine Anhänger neben ihm keine weiteren Götter haben sollen. Oder aber es wäre ausdrücklich zu unterstellen, dass lediglich der Sprecher in Strophe VII und VIII derselbe bleibt, die dargestellte Welt aber bzw. deren Grundordnung von Strophe zu Strophe sich unvermittelt ändert. Im einen wie dem anderen Falle ergäbe sich sogleich die Frage nach Ursache und Konsequenzen des jeweiligen Phänomens. Weiter zu untersuchen wäre entweder, warum und mit welcher Folge der Sprecher gegen das erste der zehn Gebote verstößt, oder andernfalls eben, wie es dazu kommt, dass aus dem jüdisch-christlichen Gotteshimmel, der die dargestellte Welt in der siebten Strophe noch überwölbt, in Strophe VIII wieder ein heidnisches Pantheon geworden ist. Da aber Wellbery keiner 
„Psalter“ (V.43) oder „Psalmen“ (V.76) gewesen sein, welche - in Konkurrenz allerdings zu den Anspielungen auf die griechisch-römische Mythologie (vgl. V.24f.: Fortunas Wagen, V.16: „Faden“ u. V.17: „Schere“ der Parzen) - an die Bibel und deren Hauptakteur denken lassen. ${ }^{10}$ Bei dieser Lesart ergibt sich jedoch die Schwierigkeit, dass Goethes Text Propositionen enthält, die mit den Objektschemata ,christlicher Gott' und ,Agape' nur schwer zu vereinen sind:

(1) Die vom „Vater der Liebe“ (V.44) gespendete Liebe kann „Balsam“ sein, kann aber ebenso gut auch als „Gift“ (V.36) wirken. Wer sie in „Fülle“ zu „trinken“ (V.38) bekommt, entwickelt weder „eine unbegrenzte Bereitwilligkeit zum Geben und zum Vergeben" ${ }^{11}$ noch erreicht er die „,völlige Überwindung des Hasses und der Rache“. ${ }^{12}$ Der reichliche Empfang dieser Liebe vermag vielmehr die gegenteilige Reaktion hervorzurufen. Er ist im Falle des Wanderers die Ursache von „Menschenhaß“ (V.37) gewesen.

(2) Dem göttlichen Adressaten wird angetragen, die „Brüder der Jagd“ (V.53) (die das Jagdprivileg besitzenden adligen „Reichen“ - V.22) mit ,jugendlichem Übermut fröhlicher Mordsucht“ (V.55) zu „segne[n]“ (V.53). Der angesprochene Gott soll Eigenschaften fördern (Übermut, Mordsucht, „Rache“-Streben), die zu christlichen Werten wie Demut und Nächsten- bzw. gar Feindesliebe in Gegensatz stehen. ${ }^{13}$

(3) Während der jüdisch-christliche Gott keine weiteren Gottheiten neben sich duldet, hat der in Strophe XII als „Du“ angeredete Gott

dieser Fragen nachgeht, wirkt seine Deutung der Textstelle unbegründet bzw. willkürlich.

10 Weimar (1984) spricht vom „Gebetston mit unüberhörbaren Anklängen an biblische Sprache“ (S. 26), Friedrich (1991) entsprechend von einem „bibelsprachlichen Anklang“"(S. 171).

11 Religion in Geschichte und Gegenwart. 2. völlig neu bearb. Aufl., Bd. 3. Tübingen 1929; Stichwort ,Liebe': Sp. 1638.

12 Ebd. Beide Zitate führen aus, inwiefern „nach Jesu Gebot [...] die Liebe der Liebe Gottes entsprechen und also vollkommen sein [soll]".

13 Sicher, der Sprecher führt an, dass die „Schwein[e]“-Jagd wohltätige Wirkung habe, insofern sie einen „Unbill“ beende, gegen den sich „mit Knütteln der Bauer [schon Jahre vergeblich wehrt]" (Strophe VIII). Durch diesen Hinweis ist jedoch das Verhalten der Jagdbrüder nur vordergründig gerechtfertigt. Dass nämlich die Bauern den Wildschäden "schon Jahre“ ausgesetzt waren, ohne sich dagegen anders als nur mit „Knütteln“ wehren zu dürfen, sagt letztlich etwas über die Verantwortunglosigkeit eines Adels aus, der sich das Jagen als Privileg vorbehält, die Jagd dann aber nicht etwa planmäßig, sondern allein zur Befriedigung persönlicher Vorlieben - nur aus „fröhlicher Mordsucht" - betreibt. 
„Brüder“ (V.87) um sich herum, so dass er - wie der griechische Zeus bzw. römische Jupiter - den Rang lediglich eines ,primus inter pares ${ }^{6}$ einnimmt.

Die Beschreibung der Liebe als eine Medizin, die bei übermäßiger Anwendung zu „Gift“ (V.36) werde, greift eine aus der antiken Philosophie bekannte Vorstellung auf: Als in Platons „Symposion“ (186a-189a) der Arzt Eryximachos an der Reihe ist, sich über den Eros zu äußern, stellt er die Heilkunde neben der Musik als eine „Wissenschaft von den Liebestrieben“ dar. ${ }^{14}$ „In der Musik sowohl als in der ärztlichen Kunst"15 komme es darauf an, eine „weise Mischung“ aus „himmlischem“ und ,gewöhnlichem“ Eros zu erreichen, so erklärt der Redner. Da „Maßlosigkeit und Unordnung in diesen Liebestrieben“ die Ursache von „Krankheiten bei Mensch und Tieren" seien, ${ }^{16}$ müsse ein tüchtiger Arzt sich darauf verstehen, „denen, die keinen Eros in sich haben, [...] ihn zu verschaffen, und denen, die ihn [zu viel, MS] in sich haben, ihn wegzunehmen" ${ }^{17}$ Erst eine rechte Dosierung von „beiderlei Eros“ ermögliche es nämlich dem Patienten, „die Begierden [...] gut zu verwenden, so daß man, ohne krank zu werden, die Lust erntet“. ${ }^{18}$ Werde dagegen der sinnliche Eros, „,wo man ihn zuführt", nicht hinreichend „vorsichtig [zugeführt]“, dann könne dieser so „manches [verderben]“ und schweren „Schaden [anrichten]“. ${ }^{19}$

Vor diesem Wissenshintergrund liegt es nahe - zumal der „Hederich“, das von Goethe gerne benutzte mythologische Lexikon, auch die in Strophe X genannte „Fackel“ zu den Attributen des antiken Liebesgottes zählt $^{20}$ - mit dem Signifikanten ,Liebe‘ eben nicht das Objektschema ,Agape ${ }^{‘}$ zu verbinden, sondern als Basis für weitere Inferenzen das kulturelle Wissen über den griechisch-römischen Eros anzusetzen.

Somit könnte man dann als den „Vater der Liebe“ (V.44) gleich eine ganze Reihe von Göttern in Betracht ziehen - bekanntlich werden dem Wesen Eros (Amor, Cupido) in den antiken Mythen vielerlei Eltern nachgesagt - und dürfte bei diesem Akteur nicht mehr länger einfach voraussetzen, dass er die Eigenschaften des gütigen Christengottes besitzt.

14 „Symposion“, 186c, zitiert nach Platon (1986), S. 125.

15 Ebd., S.127.

16 Ebd.

17 Ebd., S.125.

18 Ebd., S.127.

19 Ebd.

20 Hederich (1967), Sp. 811 u. 812. 


\section{Art der Heilmaßnahmen}

Betrachten wir nun genauer, welche therapeutischen Maßnahmen vom „Vater der Liebe“ (V.44) erwartet werden, so lässt sich feststellen, dass der Erfolg, den diese Maßnahmen verheißen, jeweils als recht zweifelhaft erscheint:

Der erste (explizite) Hilfe-Appell lautet, es möge der „Vater der Liebe“ dem Patienten, „der sich Menschenhaß aus der Fülle der Liebe trank“ (V.37f.), mit einem „Ton“ (V.44) seines „Psalter[s]“ (V.43) das Herz „erquicke[n]“ (V.46). Dass hier Musik als Heilmittel fungieren soll, muss nicht weiter überraschen, insofern ja laut Platons Eryximachos „auch die Musenkunst“ - gleich der „Heilkunde“ - „eine Wissenschaft von den Liebestrieben" ${ }^{\text {"21 }}$ ist. Nicht anders als mit den medizinischen Pharmaka, so das in Strophe VII zu inferierende Musikkonzept, vermag ein Kundiger auch mit Tönen (beiderlei) „Liebe [einzuflößen]“" ${ }^{22}$ wobei es jedoch einmal mehr wichtig ist - ,[i]n der Musik sowohl als in der ärztlichen Kunst ${ }^{\text {" } 23}$-, die bekömmliche Dosis ,vorsichtig“"24 einzuhalten. Heißt es dagegen nun aber vom „Vater der Liebe“ (V.44), er verschaffe an „Freuden“ (V.51) jedem ,ein überfließend Maß“ (V.52), so darf wohl als fraglich gelten, ob seine Liebesapplikation für den „Verächter“ (V.39) tatsächlich ein „Balsam“ (V.36) wird sein können oder ob sie auf diesen nicht doch wieder - wie schon vorher die „Fülle der Liebe“ (V.38) - als ein „Gift“ (V.36) wirken muss, das weiteren „Menschenhaß“ (V.37) schürt.

Die beiden nächsten Hilfe-Appelle sodann - „Öffne den umwölkten Blick / Über die tausend Quellen / Neben dem Durstenden / In der Wüste“ (V.47ff.), ,[...] den einsamen hüll' / In deine Goldwolken!“ (V.60f.) widersprechen einander, solange man den ersten davon als eine Aufforderung versteht, den „umwölkten Blick“ mittels ,Entwölkung“ zu „öffnen“. In diesem Falle wäre nämlich unverständlich, warum wohl der „,[E] insame“ (V.60) gleich anschließend in „Goldwolken [gehüllt]“ werden soll (V.61); hätte man ja dann doch zu unterstellen - wenn schon die Umwölkung in Strophe VIII sichtbehindernd war -, dass der soeben befreite Blick durch die gewünschte neue Wolkenhülle sofort wieder verschlossen würde.

21 „Symposion“, zitiert nach Platon (1986), S. $125 f$.

22 Ebd., S. 126.

23 Ebd., S. 127.

24 Ebd. 
Um diesen Widerspruch auflösen zu können, muss man berücksichtigen, welche Information über die Gegebenheit, Wolken' zwei weitere Textstellen vermitteln. Wird in der ersten Strophe ein „Geier“ (V.1) beschrieben, der „nach Beute schaut“ (V.4), während (und obgleich) er sich über „schweren Morgenwolken“ (V.2) befindet, und heißt es in Strophe XII dann ähnlich, das dort angeredete „Du“ (V.82) schaue ,aus Wolken“ (V.85) auf die „Reiche und Herrlichkeiten“ (V.86) der Erde, so findet sich sowohl zu Beginn als auch am Ende des Textes angedeutet, dass Wolkendecken in der hier dargestellten Welt einer bestimmten Art des Sehens - nämlich dem „Schauen“ (vgl. V.85) - keineswegs abträglich sind, so dass man als Leser sich hüten muss, mit diesem Objekt allein dessen realistische Eigenschaften zu assoziieren.

In zahlreichen Texten des Autors erscheinen Wolken, Dunst und Nebel als wandlungsfähige Projektionsfläche für „von der Einbildungskraft [...] vorgespiegelte Vision[en] “. ${ }^{25}$ Sie fungieren als ein „würdige[r] Boden“ für die „Luftbilder“ all derer, die im weitesten Sinne zum Kreise der „Imaginanten“, „Phantomisten“, „Phantasmatiker“ oder „Nebulisten“ gehören. ${ }^{26}$ Diese Motiv-Verwendung reicht wiederum bis in die griechische Klassik zurück, wo ja bekanntermaßen schon die Wolken des Aristophanes einen jeden Menschen, welcher „Lust auf böse Werke hat" ${ }^{27}$ mit vorgegaukelten Wunschbildern ${ }^{28}$ so lange in „Wahn und Illusi-

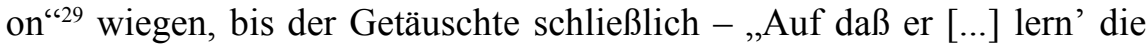
Götter fürchten“ - ,ins Unglück [...] gerät““. ${ }^{30}$

Wenn man mit einem solchen Televisions-Charakter der Wolken auch in der „Harzreise“ rechnet, so hat man sich jenen da „Durstenden“ (V.49) nicht etwa inmitten von „Quellen“ (V.48) vorzustellen, die wahrzunehmen ihn allein sein ,umwölkte[r] Blick“ (V.47) noch hindert, sondern tatsächlich als einen „Durstenden / In der Wüste“ (V.49f.), den die erwünschte Wolkenhülle darüber hinwegtäuschen soll, auf welch „öden Gefilden“ (V.70) seines Lebens Wege verlaufen.

Des Sprechers Umwölkungs-Bitte würde demnach auf eine Illusionierung zielen, die einen grundlegenden Mangel, statt ihn zu beseitigen, lediglich verdeckt. Man könnte im doppelten Sinne von einer Schein-

25 Goethe (1987): Des Epimenides Erwachen, S. 216.

26 Goethe (1988): Der Sammler und die Seinigen, S. $123 \mathrm{f}$.

27 Aristophanes (1963): Die Wolken, V.1459.

28 Vgl. ebd., V.348ff.

29 Ebd., V.819.

30 Ebd., V.1460. 
befriedigung sprechen, insofern hier einem Ungenügen mittels schönen Scheins (eben nur) scheinbar abgeholfen wird.

Voraussetzung für diese Art Lösung ist eine zum Wahn gesteigerte Einbildungskraft, wie sie gemäß platonischer Überlieferung durch die mit dem ersten Appell verlangte - Erhöhung der Liebestriebe erreicht wird, ${ }^{31} \mathrm{zu}$ der aber nach einer anderen Vorstellung auch die - als vierte Maßnahme geforderte - Bekränzung mit „Wintergrün“ (V.62) etwas beitragen kann.

Unter die als „Wintergrün“ bezeichneten Pflanzenarten fällt laut Deutschem Wörterbuch u.a. der Efeu. ${ }^{32}$ Und dieser wiederum gilt seit der Antike als eine Poeten-Droge, wie man im 18. Jahrhundert etwa aus Zedlers Universal-Lexikon wissen konnte:

Man hat auch vor Zeiten die Dicht=Meister, und Poeten mit Epheu gecrönet, damit hierdurch ihre Sinnen, die von vielem und scharffen Nachsinnen erhitzt, und fast in eine Raserey gerathen, mögen besänfftiget werden. $\mathrm{Da} ß$ also nicht unglaublich ist, daß, wie etliche sagen, in dem Winter-Grün ein Geist sey, der die Raserey erwecket, die Sinnen betäubet, und ohne Wein truncken macht. Dahero der Epheu von Dioscor. Fons Poetica genennet wird. ${ }^{33}$

Widersprüchlich wie diese Angaben zu seiner pharmazeutischen Wirkung - „,besänfftiget“ der Efeu die „Raserey“ oder „,erwecket“ er sie?! sind auch die Aussagen über des „Winter-Grüns“ sinnbildhafte Potenzen. Wegen ihrer immergrünen Beschaffenheit kann die Pflanze ,als Zeichen für den Anspruch des Dichters auf ewigen Ruhm“"34 stehen, wegen ihres zerstörerischen Schmarotzertums aber genauso auch ein Symbol der Zerstörungsgewalt und Vergänglichkeit sein: Im Hymnus „Der Wandrer“, den Goethe zwei Jahre vor der „Harzreise“ verfasste, wird der „Efeu“ neben „Moos“, „Disteln“, „Brombeergesträuch[...]“ und „hohe[m] Gras“ (!) als eines der ,Werkzeuge" beschrieben, mit denen die ,[r]eichhinstreuende Natur“ alle Kunstwerke des Menschen - ihres „Meisterstücks Meisterstück[e]“" - ,[u]nempfindlich zertrümmer[t] “. ${ }^{35}$ Die ,umkleide[nde] ${ }^{\text {“36 }}$ Pflanze ist dort Bestandteil eines Naturraums, der wie die „Öde“ des winterlichen Harzes den (einsamen) Menschen mitsamt seinen Lebenszeugnissen - den Spuren - „verschlingt“ (V.34). Von ihren Ranken „um-

31 Vgl. hierzu etwa in Platons „Phaidros“, 244a-245c.

32 Vgl. Deutsches Wörterbuch, Bd. 14.2. Leipzig 1960, Sp. 443.

33 Zedler (1961), Sp. 1361.

34 Friedrich (1991), S. 176.

35 Goethe (1985a): ,Der Wandrer“, S. 205, V.75f.

36 Ebd.: „Der Wanderer“, S. 204, V.61. 
g[eben]“ (V.62) zu werden, wie es der „Harzreise“-Sprecher als einen Liebesdienst für den „Dichter“ (V.65) erbittet, kann also einmal mehr auch zerstörerisch sein.

Nur wenig vertrauenserweckend wirkt schließlich, was die personifizierte „Liebe“ (V.65) des göttlichen Vaters in Strophe X dann tut. Über abgründige (V.69: ,grundlose“) Wege lenkt sie - lenkt der fackeltragende Eros (der als des „Chaos wunderlicher Sohn“ in Goethes Faust unter dem Namen Mephistopheles auftritt) ${ }^{37}$ - den ,in der Wüste“ (V.50) wandelnden Wanderer immer weiter durch „öde Gefilde“ (V.70), ihn hinaufführend zu jenem „gefürchteten Gipfel“ (V.78), der im kulturellen Wissen des 18. Jahrhunderts als Teufels- und Hexenplatz gilt (für „ahndende Völker“ [V.81] von „Geisterreihen“ [V.80] besucht ist). ${ }^{38}$

Diese Führung des Wanderers auf einen hohen Berg, von dessen Gipfel aus er sich die „Reiche und Herrlichkeit“ (V.86) der „Welt“ (V.84) beschauen kann, steht in intertextuellem Bezug zur biblischen Geschichte von Jesu Versuchung: Nachdem auch dort der Geführte erst in die Wüste geleitet und dann an Abgründe gebracht worden ist, nimmt ihn der Teufel „auf einen sehr hohen Berg und zeigte ihm alle Reiche der Welt und ihre Herrlichkeit“ und spricht zu ihm: „Das alles will ich dir geben, wenn du niederfällst und mich anbetest." (Mt 4, 8f.) Die Homologie zwischen beiden Erzählungen ist augenfällig: Die „Liebe“ (V.65) in Goethes Text Eros - verhält sich zum „Dichter“ (V.65) wie der christliche Teufel zum Gottessohn. Im Gegensatz aber zu Jesus, der die geforderte Anbetung verweigert, macht sich der Brockenbesteiger „des [...] Gipfels [...] Scheitel“ - Goethe als Tagebuchschreiber spricht vom „Teufels Altar“39 - zu einem „Altar des lieblichsten Danks“(V.77). - Und wofür dankt er?

\section{0. ,Therapeutische Effekte“}

Unter dem Einfluss des vom „Vater der Liebe“ (V.44) herrührenden Eros

- jener bewegenden Kraft, die nach platonisch-sokratischem Verständnis

37 Goethe (1986): Faust I, S. 572, V.1384; Goethe (1997), S. 217, V.8027. Gemäß der Theogonie Hesiods (V.120) ist Eros ein direkter Nachkomme des Chaos. Zur Personalunion von Eros und Mephistopheles vgl. Stein (2001).

38 Dass sich der überirdische ,Reiseleiter“ eines „beizenden Sturm[s]“ bedient (V.73), um den Wanderer emporzuheben, gleicht seine Beziehung zu ihm - durch die erneute Aktualisierung des schon in Strophe I aufgerufenen Skripts ,Beizjagd` - dem Verhältnis von Jäger und Beute an

39 Eintrag vom 10.12.1777, zitiert nach Weimar (1984), S. 34. 
dazu antreibt, sich durch „Zeugung im Schönen“ weitestmöglich „unsterblich" zu machen ${ }^{40}$ - ist aus dem Wanderer, der aufgrund seiner Einsamkeit verschlungen zu werden drohte, ein schöpferischer „Dichter“ (V.65) geworden: jemand, der sich mit seinen Werken das Staunen der Welt - ein (vermeintlich) dauerhaftes Andenken - hat sichern können.

Trotz dieser zentralen Zustandsveränderung wird man aber die dargestellte Geschichte - anders als der umwölkte Protagonist selbst - nicht einfach für eine ,Erfolgsstory' halten dürfen.

Von einer erfolgreichen Seelen-Behandlung wäre (unter Voraussetzung der christlichen Liebesideologie) zu erwarten, dass sie den als „Verächter“ (V.39) charakterisierten Patienten auch von seinem „Menschenhaß“" (V.37) heilt. Aber genau zu dieser Veränderung kommt es in der Geschichte nicht. Die Lebens- und Menschenferne des dargestellten Subjekts ist auf dem winterlichen Brocken größer denn je: Äußert der „Dichter" (als Sprecher des Gedichts) den Wunsch, es möge sein Lied wie ein nach „Beute“ (V.4) schauender „Geier“ (V.1) in die Welt fliegen - auf „sanften“ (V.3) Fittichen zwar, aber zur Beize doch so bereit wie in „fröhlicher Mordsucht“ (V.56) die „Brüder der Jagd“ (V.53) oder ein „beizende[r] Sturm“ (V.73) -, so lässt dies auf seine Einstellung gegenüber den „Lied“-Empfängern schließen und berechtigt wohl kaum zu der Diagnose, dass sein durch erlittene Verachtung „[zusammengezogenes] Herz" (V.72) sich wieder erweitert habe.

Sofern es sich bei dem therapierenden Arzt-Gott um den Vater der christlichen Agape handelte, müsste man diese Nicht-Heilung des Hasses als einen ereignishaften Fehlschlag des doch eigentlich unfehlbaren Christengottes ansehen. Erkennt man dagegen in der fackeltragenden Liebe „des Chaos wunderlichen Sohn“ Eros und vergegenwärtigt sich dessen Charakterisierung im Faust, ${ }^{41}$ erscheint die unveränderte Men-

40 Vgl. in Platons „Symposion“, 206b-209b.

41 Als „des Chaos [...] Sohn“ - Eros - ist in Goethes Tragödie, wie schon gesagt, Mephistopheles mehrfach ausgewiesen, dem der „Herr“ die Aufgabe zuweist, durch Reizen, Wirken und ,teuflisches' Schaffen einer ,Erschlaffung' der menschlichen Tätigkeit vorzubeugen (Goethe [1986], S. 544, V.340ff.). Gleich Goethes Prometheus oder auch dem „Luzifer“ im 8. Buch von „Dichtung und Wahrheit“ besitzt und verkörpert Mephisto eine „Schöpfungskraft“ (Goethe [1985b], S. 379), die ihn und seine ,Klienten“ zu begrenzter Produktivität befähigt. Mittels des ihm eigenen Elements, des Feuers, vermag er Kreaturen wie den Homunculus zu erschaffen: künstliche Halbwesen ohne Fleisch und Blut, denen es jeweils an wirklichem Leben gebricht. Was indes auf natürliche Weise (durch geschlechtliche Zeugung) entsteht, verachtet der Goethesche Teufel und versucht es ,,mit Wellen, Stürmen, Schütteln [und] Brand“ zu „vernichten“ (Goethe 
schenverachtung des Patienten durchaus konsequent. In diesem Falle würde das zentrale Ereignis der Geschichte darin bestehen, dass der dargestellte Dichter, um über die Vergänglichkeit triumphieren zu können, einen Bund mit dem ,Teufel' eingeht: er sich, wie später der Doktor Faust, einer misogynen „Kraft“42 überlässt, die zwar zu künstlerisch-geistiger Produktivität verhilft, gegen die natürlich gezeugte „Tier- und Menschenbrut" aber die ,kalte Teufelsfaust [...] ballt!“443

\section{Literatur}

Aristophanes

1963 Die Wolken. Übertragen von Otto Seel. Stuttgart.

Conrady, Karl Otto

1987 Goethe. Leben und Werk. Frankfurt a.M.

Dwars, Jens-F.

1998 Goethes Harzreise 1777. Spuren eines Abenteuers. Bucha bei Jena.

Friedrich, Hans-Edwin

1991 Der Enthusiast und die Materie. Von den „Leiden des jungen Werthers“ bis zur „Harzreise im Winter“. Frankfurt a.M. u.a.

Gabriel, Norbert

1992 Studien zur Geschichte der deutschen Hymne. München.

Goethe, Johann Wolfgang

1985a Sämtliche Werke nach Epochen seines Schaffens. Münchener Ausgabe, hg. von Karl Richter. Bd. 1.1. München.

1985b Sämtliche Werke nach Epochen seines Schaffens. Münchener Ausgabe, hg. von Karl Richter. Bd. 16. München.

1986 Sämtliche Werke nach Epochen seines Schaffens. Münchener Ausgabe, hg. von Karl Richter. Bd. 6.1. München.

[1986], S. 572, V.1360ff.). Entsprechend ist der von ihm repräsentierte Eros nicht einfach mit dem Geschlechtstrieb gleichzusetzen, sondern, wie schon der bei Platon diskutierte Eros, als päderastisch und misogyn zu erkennen. In Goethes Faust-Welt werden grundsätzlich - nicht nur von Mephisto selbst und nicht nur während der Grablegungs-Szene - die „allerliebsten Jungen“ (Goethe [1997], S. 341, V.11763) und „schönen Kinder“ (ebd., S. 341, V.11769) begehrt, wohingegen „Frauen-Schönheit“ in ihr ,nichts heißen [will]“ (ebd., S. 197, V.7399) und weibliche Sexualität Ächtung und Tod nach sich zieht. Von daher steht auch der „so ziemlich eingeteufelt[e]“ (Goethe [1986], S. 633, V.3372) „Wandrer“ Faust (ebd., S. 651, V.3900) vor dem Problem, dass seine Erdentage eine „Spur“ hinterlassen sollen (Goethe [1997], S. 335, V.11583), ihm eine Familiengründung aber - als die ,natürliche' Art der ,Selbstverewigung “ nicht möglich ist; vgl. dazu ausführlicher Stein (2001), S. 206-215.

42 Goethe (1986): Faust I, S. 571, V.1335.

43 Ebd., S. 572, V.1369 u. $1381 f$. 
1987 Sämtliche Werke nach Epochen seines Schaffens. Münchener Ausgabe, hg. von Karl Richter. Bd. 9. München.

1988 Sämtliche Werke nach Epochen seines Schaffens. Münchener Ausgabe, hg. von Karl Richter. Bd. 6.2. München.

1997 Sämtliche Werke nach Epochen seines Schaffens. Münchener Ausgabe, hg. von Karl Richter. Bd. 18.1. München.

Hederich, Benjamin

1967 Gruendliches mythologisches Lexikon. [Leipzig / Gledisch 1770]. Neudruck. Darmstadt.

Platon

1986 Meisterdialoge. Eingeleitet von Olof Gigon. Übertragen von Rudolf Rufener. 2. Aufl. Zürich / München.

Schönert, Jörg

1996 „Aber [...] wer ists?“ Die Referenz der Aktoren in „Harzreise im Winter“ als Deutungsproblem, in: Gerhard Sauder (Hg.): Goethe-Gedichte. Zweiundreißig Interpretationen. München, S. 90-99.

Stein, Malte

2001 „Frauen-Schönheit will nichts heißen“. Ansichten zum Eros als Bildungstrieb bei Winckelmann, Wilhelm von Humboldt und Goethe, in: Ortrud Gutjahr / Harro Segeberg (Hg.): Klassik und Anti-Klassik. Goethe und seine Epoche. Würzburg, S. 195-218.

Weimar, Klaus

1984 Goethes „Harzreise im Winter“. Zur Auslegung sprachlicher Bilder u. Rückblick und Ausblick, beides in: David E. Wellbery / Klaus Weimar (Hg.): Goethe. „Harzreise im Winter“. Eine Deutungskontroverse. Paderborn, S. 15-44 u. 82-85.

Wellbery, David E.

1984 Poetologischer Kommentar zur „Harzreise im Winter“ u. Lernerfahrungen, beides in: David E. Wellbery / Klaus Weimar (Hg.): Goethe. „Harzreise im Winter“. Eine Deutungskontroverse. Paderborn, S. 45-78 u. 79-81.

Zedler, Johann Heinrich

1961 Grosses vollständiges Universal-Lexikon aller Wissenschaften und Künste. Bd. 8. [Halle / Leipzig 1732-1754]. Neudruck. Graz. 